\title{
Concentrator Device
}

National Cancer Institute

\section{Source}

National Cancer Institute. Concentrator Device. NCI Thesaurus. Code C49883.

A device designed to increase the weight per unit volume of a substance, or in communications, a device that combines data streams in a way that they can be separated after transmission. 